\title{
Pricing Convertible Bonds Based on Black-Shcoles Formula
}

\author{
Xin $\mathrm{Du}^{1, \mathrm{a}, *}$, Lian Chen ${ }^{1, \mathrm{~b}}$ \\ ${ }^{1}$ School of Economics and Management, Nanjing University of Science and Technology, Xuanwu District, \\ Nanjing, China \\ adx_1992@foxmail.com, bllant116@yahoo.com \\ *Corresponding author
}

Keywords: Convertible Bonds, Black-Scholes Formula, Risk Neutral

\begin{abstract}
The article first introduces that convertible bonds is also a kind of financial derivatives which can be priced through using the Black-Shcoles formula, and derives PDE satisfied by convertible bond prices, then calculates the pricing formula of convertible bonds, finally, uses the formula to calculate the theoretical price of a convertible bond in the market and compare the price with the market price. The average error rate between them is $6.61 \%$, it shows that the pricing formula can accurately price convertible bonds. In the conclusion, it is taken into consideration that introducing stochastic interest rate model into the pricing equation in the future.
\end{abstract}

\section{Introduction}

Convertible bonds is a type of bond that can be converted into the stock of the company that issues the bond, when pre-agreed conditions are satisfied, and it usually has a lower coupon rate. In essence, convertible bonds are based on the common corporate bonds, and with stock option which allow purchasers to convert their purchased bonds into shares of companies that issues the convertible bonds.

Therefore, convertible bonds can be seen as a kind of financial derivative, and its target asset is the company stock that it can convert into. As financial derivative, it is very important to accurately price convertible bonds.

Because convertible bonds contain stock options, the pricing of convertible bonds can refer to the pricing of options. Among the pricing methods of options, the most famous is the Black-Scholes equation proposed by Black and Scholes (1973) ${ }^{[1]}$. This equation is a milestone in the pricing of financial derivatives, because its pricing method can be used not only for option pricing but also for the pricing of other financial derivatives, including the pricing of convertible bonds. Next, the article will use the method to price convertible bonds.

\section{Equation Derivation}

First, assume that the price $f$ of the convertible bond is a function of the stock $S$ and time $t$, so that $f=f(S, t)$. The stock price $S$ satisfies the following stochastic process:

$$
\frac{d S}{S}=\mu d t+\sigma d z
$$

Among them, $\frac{d S}{S}$ is the stock return rate, $\mu$ is the drift rate of return rate, $\sigma^{2}$ is the variance of the return rate, $z$ is the Wiener process with a drift rate of 0 and a variance ratio of 1 .

According to Itō's lemma

$$
d f=\left(\frac{\partial f}{\partial S} \mu S+\frac{\partial f}{\partial t}+\frac{1}{2} \frac{\partial^{2} f}{\partial S^{2}} \sigma^{2} S^{2}\right) d t+\frac{\partial f}{\partial S} \sigma S d z
$$

It can be seen that $d f$ contains the risk term $\frac{\partial f}{\partial S} \sigma S d z$. In order to eliminate the risk term, a combination $M$ of convertible bonds and stocks can be constructed. 
Let

$$
M=-f+\frac{\partial f}{\partial S} S
$$

and then get

$$
\Delta M=\left(-\frac{\partial f}{\partial t}-\frac{1}{2} \frac{\partial^{2} f}{\partial S^{2}} \sigma^{2} S^{2}\right) \Delta t
$$

At this time, the asset portfolio is risk-free within the time $\Delta t$, so

$$
\Delta M=r M \Delta t
$$

So

$$
\left(-\frac{\partial f}{\partial t}-\frac{1}{2} \frac{\partial^{2} f}{\partial S^{2}} \sigma^{2} S^{2}\right) \Delta t=r\left(-f+\frac{\partial f}{\partial S} S\right) \Delta t
$$

So

$$
\frac{\partial f}{\partial t}+r S \frac{\partial f}{\partial S}+\frac{1}{2} \frac{\partial^{2} f}{\partial S^{2}} \sigma^{2} S^{2}=r f
$$

(7) is the PDE satisfied by the price $f$ of the convertible bond.

The options contained in the convertible bond are American call options, according to Hongzhong Liu(2003) ${ }^{[3]}$, American call option will not be executed before the maturity date. So the holder of the convertible bond will not be executed ahead of time. The boundary condition of the convertible bond is

$$
f=\max \left(q S_{T}, F\right) \text {, when } t=T
$$

Among them, $T$ is the expiration time, $q$ is the conversion ratio, and $F$ is the face value of the bond, usually taken as 100 .

\section{Equation Solving}

With an appropriate transformation, the explicit solution of the equation can be found, which is the expression of the convertible bond price.

We can see that the above PDE does not contain any variables that are affected by investor risk preference, so we can assume that all investors are risk neutral. This means that the required return on investment by investors is the risk-free interest rate $r$.

Therefore, for the price of the initial convertible bond $\mathrm{v}$, the following formula is right:

$$
\mathrm{v}=e^{-r T} \mathrm{E}\left[\max \left(q S_{T}, F\right)\right]
$$

Through appropriate deformation, we can get:

$$
\mathrm{v}=e^{-r T} F+q e^{-r T} \mathrm{E}\left[\max \left(S_{T}-F / q, 0\right)\right]
$$

Let $G=\ln S$

$$
\frac{\partial G}{\partial S}=\frac{1}{S}, \frac{\partial^{2} G}{\partial S^{2}}=-\frac{1}{S^{2}}, \frac{\partial G}{\partial t}=0
$$

Substituting (11) into (2), get

$$
d G=\left(\mu-\frac{\sigma^{2}}{2}\right) d t+\sigma d z
$$

So

$$
d l n S=\left(\mu-\frac{\sigma^{2}}{2}\right) d t+\sigma d z
$$




$$
\ln S_{T}-\ln S_{0} \sim N\left(\left(\mu-\frac{\sigma^{2}}{2}\right) T, \sigma^{2} T\right)
$$

According to risk neutral, take $\mu=r$

$$
\ln S_{T} \sim N\left(\ln S_{0}+\left(r-\frac{\sigma^{2}}{2}\right) T, \sigma^{2} T\right)
$$

Integrate (15)

$$
\begin{array}{r}
e^{-r T} \mathrm{E}\left[\max \left(S_{T}-\frac{F}{q}, 0\right)\right]=S_{0} \varphi\left(d_{1}\right)-e^{-r T} \frac{F}{q} \varphi\left(d_{2}\right) \\
\mathrm{v}=e^{-r T} F+q S_{0} \varphi\left(d_{1}\right)-e^{-r T} F \varphi\left(d_{2}\right)
\end{array}
$$

And

$$
\begin{aligned}
& d_{1}=\frac{\ln \left(q S_{0} / F\right)+\left(r+\sigma^{2} / 2\right) T}{\sigma \sqrt{T}} \\
& d_{2}=\frac{\ln \left(q S_{0} / F\right)+\left(r-\sigma^{2} / 2\right) T}{\sigma \sqrt{T}}
\end{aligned}
$$

$\varphi(x)$ is the cumulative probability distribution function of the standard normal distribution. In this way, the expression of the convertible price is obtained.

It can be seen that $e^{-r T} F$ in the formula is the value of the bond part, and $q S_{0} \varphi\left(d_{1}\right)-$ $e^{-r T} F \varphi\left(d_{2}\right)$ is the value of the call option contained in the convertible bond. So the formula is essentially decomposing convertible bonds into bonds and options and pricing them separately. If the convertible bonds pay interest, the present value of the unpaid interest rate should also be added, and it is recorded as $c$. So there is

$$
\mathrm{v}=e^{-r T} F+q S_{0} \varphi\left(d_{1}\right)-e^{-r T} F \varphi\left(d_{2}\right)+c
$$

\section{Empirical Researching}

This paper uses "110030.SH" as an example to calculate the theoretical price of the convertible bonds from 2018/1/2 to 2018/1/31 by using the above formula, and compares them with market prices.

Table 1 Results of empirical researching

\begin{tabular}{|c|c|c|c|c|}
\hline Date & Stock Price & Theoretical Price & Market Price & Error Rate \\
\hline $2018 / 1 / 2$ & 5.77 & 100.4184 & 105.363 & $4.92 \%$ \\
\hline $2018 / 1 / 3$ & 5.79 & 100.4822 & 105.4189 & $4.91 \%$ \\
\hline $2018 / 1 / 4$ & 5.80 & 100.5140 & 105.5548 & $5.02 \%$ \\
\hline $2018 / 1 / 5$ & 6.15 & 101.7072 & 109.2907 & $7.46 \%$ \\
\hline $2018 / 1 / 8$ & 6.27 & 102.1481 & 109.6984 & $7.39 \%$ \\
\hline $2018 / 1 / 9$ & 6.41 & 102.6831 & 109.1142 & $6.26 \%$ \\
\hline $2018 / 1 / 10$ & 6.30 & 102.2610 & 108.5601 & $6.16 \%$ \\
\hline $2018 / 1 / 11$ & 6.23 & 101.9997 & 107.596 & $5.49 \%$ \\
\hline $2018 / 1 / 12$ & 6.26 & 102.1112 & 107.2019 & $4.99 \%$ \\
\hline $2018 / 1 / 15$ & 6.24 & 102.0366 & 107.4096 & $5.27 \%$ \\
\hline $2018 / 1 / 16$ & 6.80 & 104.2846 & 110.9055 & $6.35 \%$ \\
\hline $2018 / 1 / 17$ & 6.42 & 102.7222 & 110.2314 & $7.31 \%$ \\
\hline $2018 / 1 / 18$ & 6.45 & 102.8402 & 110.2973 & $7.25 \%$ \\
\hline $2018 / 1 / 19$ & 6.35 & 102.4514 & 109.8832 & $7.25 \%$ \\
\hline $2018 / 1 / 22$ & 6.34 & 102.4131 & 109.8008 & $7.21 \%$ \\
\hline $2018 / 1 / 23$ & 6.43 & 102.7613 & 111.5167 & $8.52 \%$ \\
\hline $2018 / 1 / 24$ & 6.37 & 102.5282 & 111.4326 & $8.68 \%$ \\
\hline $2018 / 1 / 25$ & 6.30 & 102.2610 & 110.8685 & $8.42 \%$ \\
\hline $2018 / 1 / 26$ & 6.51 & 103.0784 & 112.1844 & $8.83 \%$ \\
\hline $2018 / 1 / 29$ & 6.57 & 103.3210 & 109.6721 & $6.15 \%$ \\
\hline
\end{tabular}




\begin{tabular}{|l|l|l|l|l|}
\hline $2018 / 1 / 30$ & 6.66 & 103.6916 & 109.2579 & $5.37 \%$ \\
\hline $2018 / 1 / 31$ & 6.55 & 103.2399 & 109.7438 & $6.30 \%$ \\
\hline
\end{tabular}

The stock price is the closing price of the corresponding date. According to the terms of the convertible bond, the conversion price is 7.24 . The risk-free interest rate is $3.82 \%$, the stock annual volatility is calculated to be 0.2922 , face value of 100 , maturity date of 2 years, one-year interest of 1.5 and biennial interest of 2 . The results are shown in the Table 1 and Figure 1.

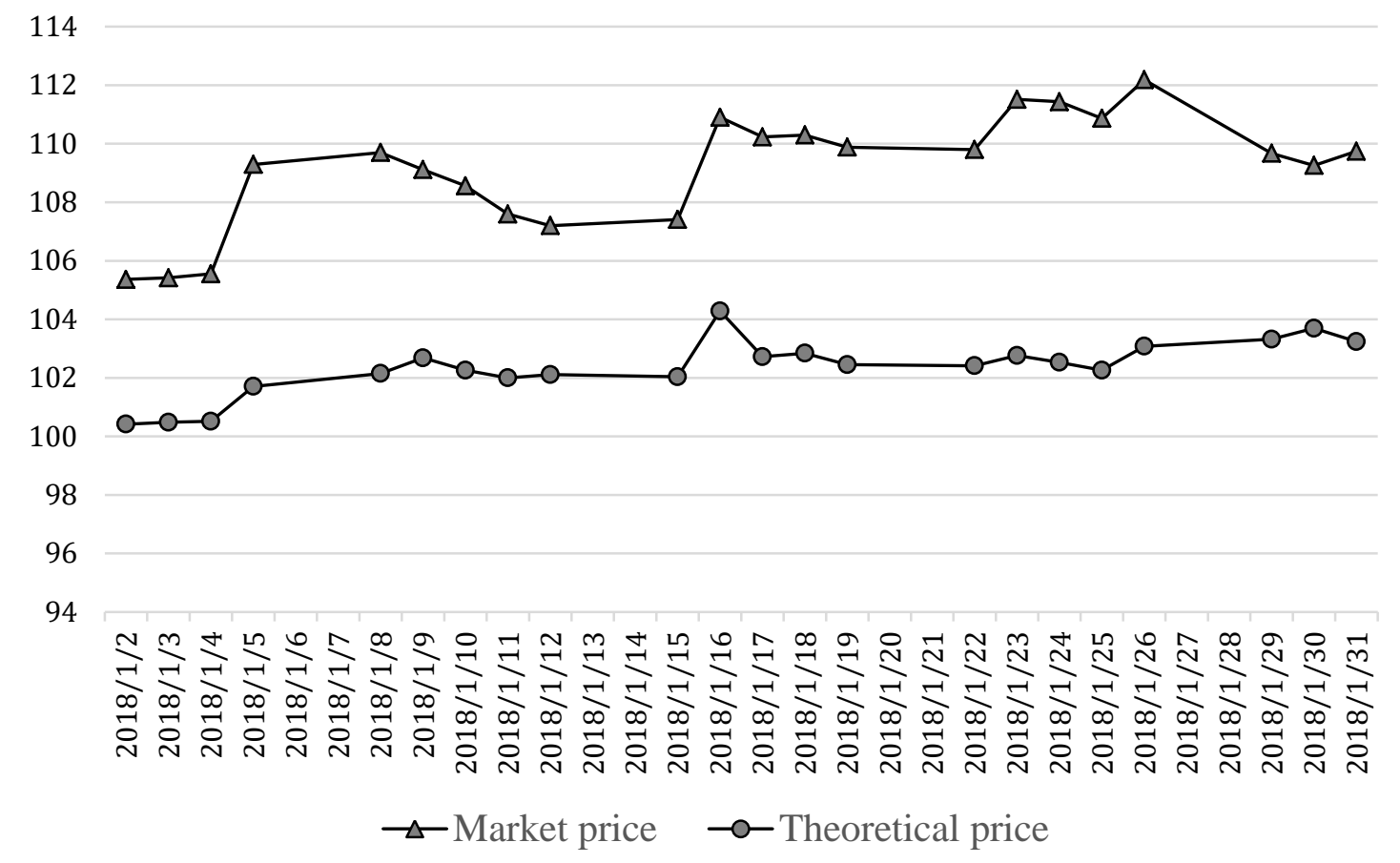

Figure 1 Market price and theoretical price

\section{Conclusion}

It can be seen that there is a certain gap between the market price and the theoretical price, resulting in a variety of reasons for the error, such as the error caused by the volatility estimation, the model setting error and the error caused by the risk-free interest rate fluctuation. The model can be further modified, for example taking into account the effects of interest rate fluctuations, and introducing the stochastic interest rate model into the pricing formula of the convertible bond.

The average error rate calculated is $6.61 \%$, and the error rate is small, indicating that this formula can accurately price convertible bonds.

\section{References}

[1] Black F, Scholes M. (1973) The Pricing of Options and Corporate Liabilities. Journal of Political Economy, 81, 637-659.

[2] Brennan M.J., Schwartz E.S. (1980) Analyzing convertible bonds. Journal of Financial and Quantitative Analysis, 15(4), 907-929.

[3] Hongzhong Liu. (2003) Investments. Higher Education Press, 288-289.

[4] Feng Ye. (2004) The Study of Pricing China’s Convertible Bonds. Fudan University.

[5] Qi'nan Lai, Changhui Yao and Zhicheng Wang. (2005) Empirical Study on the Pricing of Convertible Bonds in China. Journal of Financial Research, 9, 105-121.

[6] Qiyuan Zhou. (2007) Research on Pricing and Analyzing Convertible Bonds. Shanghai Jiao Tong University. 
